\title{
Democratizar la gobernanza digital
}

escolar: resultados de una investigaciónacción

Democratize digital school governance: action research results

Democratizar a governança digital escolar: resultados de uma pesquisa-ação
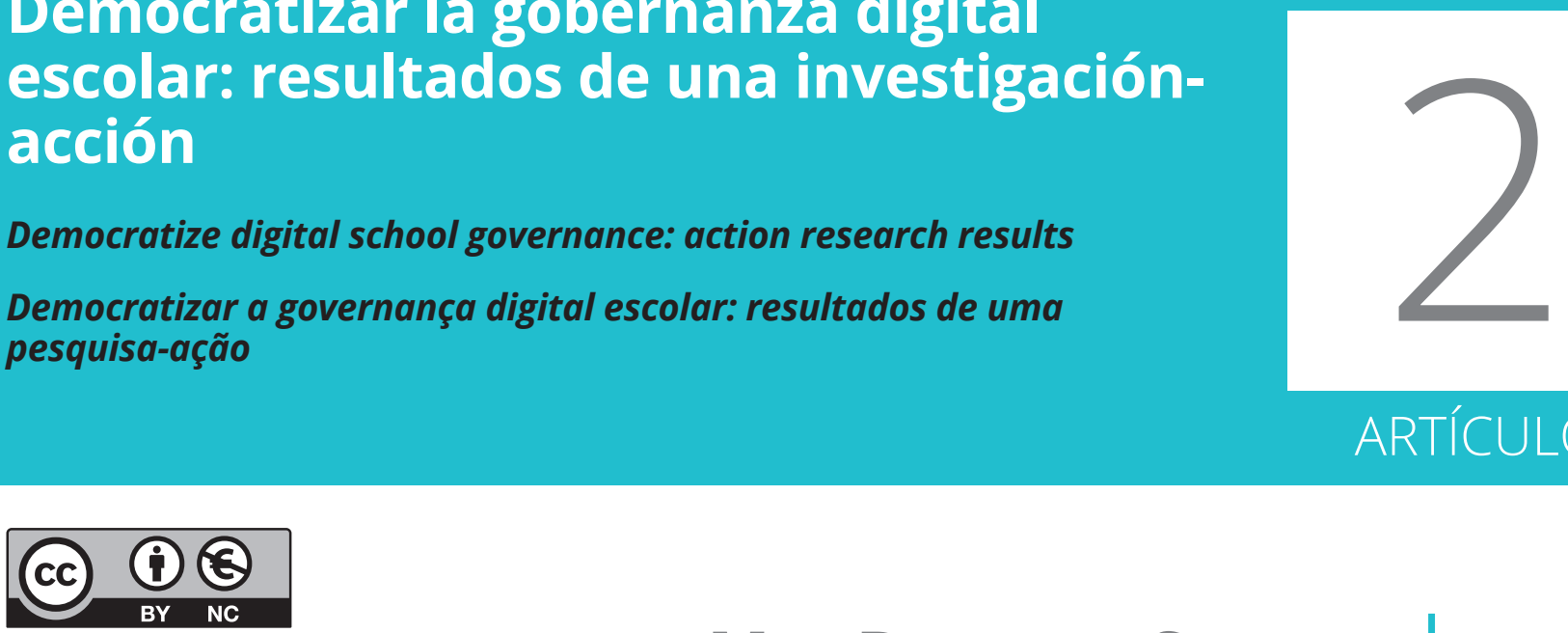

\section{Mar Beneyto-Seoane}

Universitat de Vic - Universitat Central de Catalunya (España)

La Dra. Mar Beneyto-Seoane es profesora asociada del Departamento de Pedagogía de la Universidad de Vic-UCC. Actualmente imparte docencia en el Grado de Maestro y el Grado de Educación Social. Sus ámbitos de estudio e investigación principales son el estudio de las desigualdades, la inclusión y la participación digital en el ámbito educativo y social.

mar.beneyto@uvic.cat

orcid.org/0000-0001-5946-2670

\section{Jordi Collet-Sabé}

Universitat de Vic - Universitat Central de Catalunya (España)

El Dr. Jordi Collet-Sabé es profesor titular de Sociología de la Educación en la Universidad de Vic-UCC, donde des de 2019, ejerce como vicerrector de Investigación. Ha realizado investigaciones y ha publicado a nivel internacional sobre políticas educativas; relaciones escuela, familia y comunidad: equidad educativa; socialización familiar y democracia. Ha sido Visiting Fellow en el Institute of Education - University College London.

jordi.collet@uvic.cat

orcid.org/ 0000-0001-8526-9997

RECIBIDO: 09 de enero de 2020 / ACEPTADO: 05 de mayo de 2020

\section{Resumen}

El presente artículo presenta los resultados de una tesis doctoral que analiza la tecnología digital escolar en clave democrática, y desarrolla una investigación-acción para promover la participación, la inclusión y reducir las desigual- dades en este ámbito. La investigación se ha centrado en pensar, diseñar y construir prácticas digitales más democráticas en una escuela catalana diversa, con la finalidad de reducir la brecha digital que existe entre los distintos miembros de la comunidad educativa y pro- 
mover más y mejor participación e inclusión. En este artículo mostramos el impacto que la incorporación de la tecnología digital escolar desde esta perspectiva democrática genera en la gobernanza del centro educativo. Principalmente, los resultados nos muestran que esta perspectiva genera más espacios de participación digital y mejora las relaciones que se establecen en estos espacios.

\section{PALABRAS CLAVE:}

Desigualdad social, Democracia, Tecnología de la educación, Participación, Relación padres-escuela.

\begin{abstract}
This article presents the results of a doctoral thesis that analyzes the digital school technology from a democratic perspective and develops an action research to promote the participation, inclusion and reduction of inequalities in this field. The research has focused on thinking, designing and building more democratic digital practices in a diverse Catalan school with the aim of reducing the digital gap that exists between the different members of the educational community and promoting more and better participation and inclusion. In this paper, we show the impact that the incorporation of school digital technology from this democratic perspective generates in the governance of the educational center. Mainly, the results show that
\end{abstract}

this perspective generates more spaces for digital participation and improves the relationships established in these spaces.

\section{KEYWORDS}

Social inequality, Democracy, Educational technology, Participation, Family-school relationship.

\section{Resumo}

Este artigo apresenta os resultados de uma tese de doutorado que analisa a tecnologia digital democrática da escola e desenvolve uma pesquisa-ação para promover a participação, a inclusão e reduzir as desigualdades nesse campo. A pesquisa concentrou-se em pensar, projetar e construir práticas digitais mais democráticas em uma escola catalã diversa, com o objetivo de reduzir o fosso digital existente entre os diferentes membros da comunidade educacional e promover mais e meIhor participação e inclusão. Neste artigo, mostramos o impacto que a incorporação da tecnologia digital escolar a partir dessa perspectiva democrática gera na governança do centro educacional. Principalmente, os resultados mostram que essa perspectiva gera mais espaços para a participação digital e melhora as relações que se estabelecem nesses espaços.

\section{PALAVRAS-CHAVE}

Desigualdade social, Democracia, Tecnologia educacional, Participação, Relação pais-escola.

\section{INTRODUCCIÓN}

La incorporación de la tecnología digital ha agitado las prácticas y las dinámicas cotidianas de las escuelas (Bosco et al., 2016). No solo en lo que concierne a la dimensión material (ubicación del aula de informática, el carro de ordenadores, las pizarras digitales interactivas en las aulas, entre otros ejemplos), la dimensión temporal (dedicación de cierto tiempo lectivo al aprendizaje vinculado a las tecnologías digitales) o aquello relacionado con el contenido (incorporación de la competencia digital en el currículum escolar), sino también en aquello más oculto e invisible: las relaciones interpersonales entre los distintos actores educativos (Albar, 1996; Castells, 2003). Ante esta situación, una revisión de la literatura científica nos muestra un persistente y creciente interés sobre la naturaleza (qué) y la organización (cómo) de las rela- 
ciones que se producen en el contexto escolar con el uso de la tecnología digital (Adell \& Castañeda, 2015; Beneyto-Seoane \& Collet-Sabé, 2016; Beneyto-Seoane \& Collet-Sabé, 2018; Beneyto-Seoane et al., 2013; Bosco et al., 2016; Cobo, 2017; Fullan, 2013; Selwyn, 2011, 2016). Y sobre cuáles son sus efectos (esperados y no esperados) en términos educativos, relacionales, democráticos e inclusivos.

El presente artículo se añade a las investigaciones que buscan describir, comprender y mejorar cómo son las relaciones digitales en el contexto educativo desde una perspectiva democrática e inclusiva (Baena et al., 2020). Específicamente se centra en el impacto que podría generar adoptar una perspectiva democrática ante las desigualdades digitales escolares. Para hacerlo, parte del marco teórico, la metodología y los resultados de una tesis doctoral relativa a la tecnología digital escolar en clave democrática, en la cual se desarrolla una investigación-acción sobre participación y desigualdades digitales escolares.

\section{DESIGUALDAD DIGITAL Y DEMOCRACIA ESCOLAR}

Hablar de relaciones digitales en el contexto escolar implica atender a las relaciones de desigualdad digital que existen en la escuela. Pero ¿qué entendemos por desigualdad digital en el contexto escolar? Para conceptualizar este término, partimos de dos referentes en el campo de la investigación digital y educativa. Por un lado, la perspectiva sociológica del Sistema Relacional de la Brecha Digital (Van Deursen \& Van Dijk, 2010; Van Dijk, 2012, 2005; Van Dijk \& Van Deursen, 2014). Y, por otro lado, las Dimensiones de la Democracia Escolar (Feu et al., 2017; Feu et al., 2016).

En cuanto al Sistema Relacional de la Brecha Digital, Van Dijk (2005) expone que "the point of departure of this notion of inequality is neither the essences of individuals nor the essences of particular collectives or systems but the bonds, relationships, interactions, and transactions between people" (p.11). En este sentido el sistema relacional se define como una estructura

que vincula diferentes elementos que condicionan, determinan o influyen en la desigualdad digital. Para este autor, esta perspectiva conlleva dos ventajas, por un lado, que la desigualdad digital no reside únicamente en las características particulares de cada individuo, "this kind of explanation will unearth more of the actual mechanisms creating inequality than will an explanation in terms of individual attributes" (Van Dijk, 2005, p. 12). Y, por otro lado, que permite diferenciar varios tipos de desigualdad, ya que esta entiende que la desigualdad está creada según como las estructuras de la sociedad valoran y posicionan las características individuales de las personas: "the social recognition of differences and the structural aspects of society refer to the relatively permanent and systemic nature of the differentiation called inequality" (Van Dijk, 2005, p. 12).

El sistema relacional propuesto por Van Dijk se caracteriza por cuatro elementos: las características categóricas (personales -sexo, edad, origen de nacimiento, competencias y personalidad-y de posición -laboral, familiar, nacionalidad y educación-), la distribución de recursos (relaciones de poder con los recursos: materiales, temporales, mentales, sociales y culturales), el acceso a las tecnologías digitales (relativo a la motivación, material o físico, habilidades y usos) y la participación en la sociedad (como las personas participan digitalmente en la sociedad). Y el vínculo que establece entre estas cuatro dimensiones permite describir como se genera y estructura la desigualdad digital: 
1. Las desigualdades estructurales de la sociedad (derivadas del valor que se adjudica a las características categóricas) producen una distribución desigual de los recursos.

2. Una distribución desigual de los recursos provoca un acceso desigual a las tecnologías digitales.

3. El acceso desigual a las tecnologías digitales también depende de las características de estas tecnologías.

4. El acceso desigual a las tecnologías digitales provoca una participación desigual en la sociedad.

5. La participación desigual en la sociedad refuerza las desigualdades estructurales y las distribuciones desiguales de recursos.

Ante este sistema de desigualdad, el mismo autor considera que el ámbito escolar es uno de los más importantes para llevar a cabo prácticas que disminuyan la brecha digital, tanto en la propia escuela, como para que influya en el futuro en el contexto de la sociedad.

En relación con las Dimensiones de la Democracia Escolar (Feu et al., 2017; Feu et al., 2016) es una perspectiva que propone cuatro dimensiones para identificar y definir las prácticas democráticas escolares. Estas dimensiones (Feu et al., 2013, pp. 4-6) son:

- Gobernanza: se refiere a las estructuras y a los procedimientos a través de locuales se toman las decisiones políticas y se gestiona lo público; a un método y a unas reglas de convivencia.

- Habitabilidad: la participación política en libertad e igualdad no es solo una cuestión formal, sino también material; preocupación y respuesta por las condiciones en que viven las personas; que la gobernanza necesita de unas condiciones básicas de calidad de vida y bienestar para todas las personas.
- Alteridad: es el reconocimiento de aquellos y de aquello que no es como "nosotros": del diferente, del extranjero, de la persona vulnerable, del colectivo minoritario, del que sufre, del que tiene otro sexo u otra sexualidad o capacidad, etc. También del que no es humano porque es animal, planta, naturaleza, paisaje.

- Éthos: definido como una forma de ser y estar en el mundo y con los otros, que constituye una dimensión básica de las tres anteriores [...] los valores y virtudes formaban parte de un éthos que se manifiesta de forma transversal en las tres dimensiones.

El motivo que nos lleva a utilizar esta perspectiva de democracia escolar para analizar las desigualdades digitales es que existe un estrecho vínculo con el sistema relacional que hemos expuesto anteriormente. Un primer ejemplo de ello es que hay una familiaridad entre los sistemas de relación de poder (los cuales deciden como es la distribución de recursos) y los órganos de poder (gobernanza). Un segundo ejemplo lo encontramos en que el acceso a la tecnología digital (ya sea material o temporal) está relacionado directamente con las condiciones básicas de calidad vida (habitabilidad). Y un último ejemplo es que atender al sistema de desigualdad implica reconocer a los otros, que no son como "nosotros" (alteridad). En este sentido, si sumamos el sistema relacional de la brecha digital a la perspectiva democrática escolar podemos, a la vez, visibilizar, analizar y proponer respuestas a la desigualdad digital que encontramos en los centros educativos y entre sus actores (docentes, alumnado, sus familias y personal de administración y servicios -PAS-). Por un lado, porque contempla los elementos que condicionan y estructuran la desigualdad digital (Van Deursen \& Van Dijk, 2010; Van Dijk, 2012, 2005; Van Dijk \& Van Deursen, 2014). Y, por otro lado, porque los contempla 
desde una perspectiva democrática e inclusiva que busca superar esas desigualdades (Feu et al., 2017, 2013, 2016).

Partiendo de esta doble aproximación teórica, una de las cuestiones de la investigación fue ¿qué impactos en la inclusión y la participación podría generar la incorporación de la perspectiva digital democrática en la gobernanza escolar de un centro educativo?

Para dar respuesta a esta cuestión a continuación, exponemos cuales fueron los procesos de toma de decisiones que se llevaron a cabo desde el centro en relación con los órganos escolares, como también vamos a describir los cambios e impactos que suscitó la perspectiva digital democrática en los órganos de participación (pre y post investigación). Este proceso lo vamos a describir teniendo en consideración la perspectiva y la experiencia de los diferentes miembros de la comunidad escolar: docentes, alumnado, familias y personal de administración y servicios (PAS).

\section{METOdOLOGÍA: LA INVESTIGACIÓN-ACCIÓN}

La investigación optó desde un inicio por la investigación-acción (Cohen et al., 2011; Elliott, 1993; Ferrance, 2000; Lewin, 1946; Stenhouse, 1993; Ulvik et al., 2017), por ser una metodología en la que, a partir de la colaboración, la participación y la toma de decisiones de los diferentes miembros de una misma comunidad educativa, se introducen cambios para la mejora escolar. En la investigación esta metodología implicó un proceso de planificación, actuación, observación y autorreflexión sobre una situación; y se realizó a través de la participación activa del investigador/a y de las personas involucradas en la situación (Bartolomé, 1992; Elliott, 1993; Kemmis \& MacTaggart, 1988a; Kemmis \&
McTaggart, 1988b; Latorre, 2003; McNiff et al., 2003; Ulvik et al., 2017; Beneyto-Seoane et al. 2019). Concretamente, la investigación-acción se centró en: conocer la realidad digital y de participación en un contexto escolar concreto; acompañar a su comunidad educativa en la incorporación y uso de la tecnología digital escolar desde una perspectiva democrática; y conocer el impacto de las prácticas digitales incorporadas desde esta perspectiva en las desigualdades digitales, la inclusión educativa y la gobernanza escolar.

En relación con las herramientas de recogida de datos, en la investigación-acción se utilizaron: la entrevista, los grupos de discusión, los cuestionarios y el análisis de documentos (Bisquerra, 2004; Cohen et al., 2011; Quintanal \& García, 2012; Rodríguez et al., 1999).

Por lo que se refiere al contexto, la investigación se desarrolló en un centro escolar de titularidad pública de un municipio de 45.000 habitantes de la provincia de Barcelona, que oferta desde el de segundo ciclo de la educación infantil hasta la etapa de educación primaria (escuela de unos 450 alumnos, unas 350 familias y un equipo docente de 30 maestros). El perfil de las familias del centro se caracteriza por una gran diversidad cultural (un $60 \%$ de ellas ha nacido fuera de España); el nivel de estudios es medio bajo (un 36,4\% solo han superado los estudios de primaria y un 39\% los estudios de secundaria); y en el ámbito profesional-laboral, el 72,6\% de las familias ocupan puestos de trabajo no especializados. Además, la ubicación periférica de la escuela en relación con el centro de la ciudad hace que se encuentre lejos de la mayoría de los servicios públicos del municipio como la biblioteca, la universidad, las diferentes oficinas municipales, los museos, el centro de arte, etc.

Las razones que nos llevaron a elegir este centro principalmente fueron, por un lado, la faci- 
lidad de acceso a la información y al desarrollo del estudio y, por otro lado, las características propias de la escuela (diversa y con perfiles familiares desiguales; con una clara voluntad de mejorar su práctica educativa, e implicada en redes de innovación educativa, también digitales).

Respecto al proceso de recogida y análisis de datos de la investigación-acción se distinguen 4 fases principales: preliminar, inicial, central $y$ final.

- La fase preliminar o de planificación de la investigación se llevó a cabo en el curso 2014 15, y consistió principalmente, por un lado, en la búsqueda bibliográfica sobre el tema principal de la investigación para conocer el estado actual de la cuestión sobre tecnología digital escolar y, por otro lado, en establecer el primer contacto con el centro.

- En la fase inicial o de actuación (curso 201516), en primer lugar, se realizó la primera recogida de datos (los primeros grupos de discusión, entrevistas, cuestionarios para familias, alumnado, docentes y PAS sobre el estado inicial de la cuestión), lo cual permitió reconocer la situación inicial del centro en relación con la gobernanza escolar. En segundo lugar, se realizó un primer análisis de datos para compartir los principales resultados con los docentes. Y, en tercer lugar, los docentes reflexionaron, debatieron y tomaron decisiones sobre qué acciones debían desarrollar a partir de ese momento conforme a los órganos de toma de decisiones y su funcionamiento.

- En la fase central o de observación, durante el 2016-18, se observaron las diferentes actuaciones realizadas en el centro escolar vinculadas a la gobernanza escolar con la intención de recoger datos, evaluar y cambiar dinámicas si era necesario. Durante este proceso de observación, surgieron nuevas nece- sidades y limitaciones, por lo que se desarrollaron nuevas actuaciones (nuevas recogidas de datos -grupos de discusión, entrevistas, cuestionarios y observaciones- y diseño de actuaciones).

- Por último, en la fase final o de reflexión, que tuvo lugar desde mediados del curso 2017 18 hasta mediados del 2019, se realizó una segunda recogida de datos finales y se analizaron los resultados, con el fin de evaluar el impacto del proyecto en la gobernanza escolar. Pese a que ubicamos el periodo de reflexión en esta fase final, queremos destacar que se han producido situaciones de reflexión a lo largo de todo el proceso de investigación, situación que ha permitido mejorar las actuaciones incorporadas o detectar la necesidad de planificar nuevas actuaciones. Esta reflexión constante es la que ha dado el carácter cíclico al estudio, característica propia de una investigación-acción.

La investigación-acción ha recogido y analizado información a partir de cinco contextos de la comunidad escolar: el alumnado, sus familias, el equipo docente, PAS, y la documentación del centro.

- En relación específicamente con el alumnado, se han recogido datos iniciales y finales de los alumnos de quinto y sexto grado de primaria, a través de grupos de discusión. En total han participado aproximadamente 100 alumnos. Se ha dividido cada grupo-clase en dos subgrupos y con cada subgrupo se han realizado dos grupos de discusión (inicial y final), llevando a cabo un total de 8 grupos de discusión.

- Respecto a las familias, en la primera recogida de datos se han obtenido 134 respuestas a los cuestionarios de las familias, y 236 en la segunda recogida. Además de los cuestionarios se han realizado dos grupos de discusión 
(inicial y final para cada grupo) con las familias que pertenecen a: la Asociación de Madres y Padres de Alumnos (AMPA), las familias que pertenecen a las "Mares d'Enllaç" y las familias delegadas del grupo de WhatsApp.

- Por lo que respecta a los docentes, se contestaron 30 cuestionarios en la primera fase de recogida de datos y 22 en la segunda recogida. Además, se realizaron tres grupos de discusión con todo el claustro de maestros, 11 reuniones de seguimiento con la jefa de estudios, tres grupos de discusión con los coordinadores del ciclo educativo (infantil y primaria) y dos entrevistas con los coordinadores TAC.

- En cuanto al PAS, se han realizado dos entrevistas (inicial y final) con la administrativa de la escuela, y dos grupos de discusión (inicial y final) con el personal trabajador del comedor escolar.

- Por último y con relación a la documentación, se ha llevado a cabo un análisis documental de los principales documentos de centro (Proyecto Educativo de Centro, Normas de Organización y Funcionamiento de Centro, y Proyecto TAC), como también de las principales plataformas digitales que utiliza la escuela (web y blogs de ciclo).

Esta estructura metodológica ha permitido recoger datos en torno la tecnología y las desigualdades digitales de un centro escolar concreto y, centrándonos en la finalidad de este artículo, sobre el impacto que su incorporación ha generado en la gobernanza escolar.

\section{IMPACTO EN LA} GOBERNANZA ESCOLAR: ANÁLISIS DE RESULTADOS

En este apartado, exponemos el impacto de la investigación-acción en la gobernanza esco- lar. Los resultados los hemos dividido según la definición de gobernanza de Feu et al. (2013): según la estructura u órganos de participación (espacios virtuales donde se puede participar); y según los procedimientos o relaciones que se establecen en estos órganos.

\subsection{IMPACTO EN LA ESTRUCTURA Y LOS ÓRGANOS DE PARTICIPACIÓN DIGITAL}

Como apuntábamos anteriormente, hablar de los órganos de participación significa hablar de las estructuras o espacios donde las personas se encuentran, hablan, participan, colaboran, toman decisiones y actúan. Los resultados nos muestran que la incorporación de la perspectiva democrática en la dimensión digital de la escuela, sobre todo, ha promocionado la creación de nuevas estructuras y órganos digitales de participación.

A continuación, presentamos las distintas estructuras que se crearon a partir de los primeros resultados de la investigación-acción.

\subsection{1. ÓRGANOS DE PARTICIPACIÓN DIGITAL DE LOS DOCENTES}

- Vídeo online sobre el proceso de enseñanza y aprendizaje de las matemáticas en la escuela: esta actuación nació del interés de los docentes de facilitar un recurso a las familias para que éstas pudieran acompañar a sus hijos e hijas en la realización de las tareas escolares vinculadas a las matemáticas.

- Formación de claustro sobre tecnología digital escolar para la participación: en los primeros resultados se detectó un interés de los docentes en mejorar sus competencias digitales en tecnología escolar para la participación. Recogiendo esta necesidad, el claustro 
realizó una formación que les permitió añadir prácticas digitales más participativas en las aulas.

- Creación de un blog del ciclo de educación infantil: esta actuación nace de la necesidad de las familias del ciclo de educación infantil de conocer más sobre la cotidianidad escolar de sus hijos e hijas y del interés de los docentes en mostrar su trabajo a las familias. Los docentes de esta etapa educativa decidieron crear un blog donde mostrar a las familias las actividades que se llevan a cabo en el centro.

- Revisión de las normas del grupo de WhatsApp de maestros y maestras: en los primeros resultados se detectaron ciertas incoherencias en el uso del grupo de WhatsApp de los docentes. A partir de estos resultados, los maestros decidieron tener dos grupos de WhatsApp: uno para informaciones importantes comunicadas por el equipo directivo o desde las distintas coordinaciones, y otro más lúdico donde compartir imágenes, frases, felicitaciones, entre otros temas.

- Elaboración de un documento de acogida TIC para los docentes de nueva incorporación: los docentes expusieron que sentían ciertas limitaciones al acoger a los docentes de nueva incorporación en el centro. Concretamente, en el momento de compartir los recursos digitales del centro. Por este motivo, decidieron crear un documento donde se explicitan los recursos digitales del centro, su funcionamiento y su organización.

- Notas informativas en formato digital: los docentes detectaron que las notas informativas en papel dirigidas a las familias, en numerosas ocasiones no llegaban a su destino. Por este motivo, decidieron reforzar estas notas y enviarlas también a través del correo electrónico.

\subsection{2. ÓRGANOS DE PARTICIPACIÓN DIGITAL DEL ALUMNADO}

- Elaboración de vídeos de YouTube donde el alumnado hace recomendaciones de libros: esta propuesta nació del interés que el alumnado tenía en utilizar la plataforma virtual de vídeos de Youtube. Los docentes recogieron este interés e incorporaron el uso de esta plataforma en la asignatura de lengua catalana (los alumnos hacían recomendaciones de libros a través de dicha plataforma).

- Formación del alumnado de sexto sobre seguridad en internet: en los primeros resultados, el alumnado mostró cierto desconocimiento sobre peligros y seguridad en internet (sobre todo aquellos alumnos con familias de origen inmigrado, un nivel de estudios bajo y con un posicionamiento laboral también bajo). Además, los docentes detectaron ciertas situaciones de riesgo. Por este motivo, el profesorado decidió llevar a cabo una formación con los alumnos de sexto sobre seguridad en internet.

\subsection{3. ÓRGANOS DE PARTICIPACIÓN DIGITAL DE LAS FAMILIAS}

- Vinculación de las clases de lengua catalana a la tecnología digital escolar: a partir del primer análisis, se detectó que las familias que tenían menos competencia lingüística en catalán eran las que también mostraban menos competencia digital. Esta situación conllevaba que estas familias también fueran las que menos consultaban los espacios web de la escuela. Por este motivo, los docentes decidieron impartir las clases de catalán utilizando la web de la escuela (las familias de- 
bían resolver problemas buscando la información en la web escolar).

- Creación de un grupo de WhatsApp para familias: en la fase inicial las familias mostraron un alto interés en mejorar la comunicación con la escuela, por este motivo propusieron crear un grupo de WhatsApp. En este grupo se añadió a las familias delegadas de cada curso y a la jefa de estudios. En este grupo compartían informaciones relevantes de la escuela (salidas, avisos de última hora, aspectos organizativos, recordatorios, entre otros ejemplos).

- Entrada en funcionamiento del correo electrónico para la comunicación entre familias y escuela: a partir del mismo interés detectado en la actuación anterior (mejorar la comunicación entre familias y escuela), los docentes decidieron crear un correo electrónico para comunicarse con las familias. De este modo, las familias en vez de llamar a la escuela (por ejemplo, para informar de una ausencia) podían enviar un correo (informando de la misma).

\subsection{4. ÓRGANOS DE PARTICIPACIÓN DIGITAL DEL PAS}

- Elaboración de un lipdub sobre el comedor escolar: a través de los primeros resultados se observó que unos de los agentes escolares más invisible en los espacios digitales era el personal del comedor. Con la intención de dar visibilidad al servicio y al personal del comedor, estos elaboraron un lipdub con la intención de mostrarlo a la comunidad escolar.

- Creación de un apartado en el web de la escuela donde se describen recetas de las cocineras: también a partir de detectar la invisibilidad del personal de comedor en los espacios virtuales de la escuela, los docentes crearon un espacio en el web escolar donde las cocineras proponían recetas de cocina o explicaban como es el día a día en el comedor de la escuela.

En los distintos espacios que acabamos de presentar, podemos observar como dimensiones como el acceso -de motivación, material, habilidades y usos- (Van Dijk, 2005) o la habitabilidad - crear las condiciones para la participación(Feu et al., 2013) están presentes, por ejemplo: se fomenta el acceso a la tecnología, se recogen los intereses y motivaciones para diseñar una tecnología digital más ajustada a las necesidades e intereses escolares, y se reconocen los conocimientos de los distintos miembros para mejorar sus competencias digitales, entre otros ejemplos.

A partir de los espacios y órganos que acabamos de exponer, podemos dibujar el siguiente apartado, vinculado a los procedimientos y las relaciones que se establecen en estos órganos de participación.

\subsection{IMPACTOS EN LOS PROCEDIMIENTOS Y LAS RELACIONES}

Cuando hablamos del impacto en los procedimientos y relaciones desde una perspectiva digital democrática, lo hacemos en referencia a las transformaciones que se han dado en los objetos de discusión -sobre los aspectos sobre los cuales se toman decisiones-y en la toma de decisiones -sobre las relaciones de poder que se establecen cuando se participa en entornos tecnológicos- (Feu et al., 2013) que se dan en los espacios de participación. Como también nos referimos a las relaciones de poder, la distribución de recursos o al reconocimiento del otro para el acceso digital (Van Dijk, 2005).

En relación con los espacios de participación y la toma de decisiones, a partir de los resultados 
recogidos en la fase final y de reflexión (201718), observamos que las personas involucradas en los espacios de participación principalmente han sido todos los agentes escolares (docentes, alumnado, familias y personal no docente). No obstante, quienes ha tomado casi todas las decisiones han sido los docentes. Estos agentes han sido los principales encargados de pensar, diseñar, construir y desarrollar las actuaciones de la investigación-acción. A continuación, exponemos concretamente como han sido las relaciones establecidas a partir de la investigación.

\subsubsection{RELACIONES DE PARTICIPACIÓN DIGITAL DE LOS DOCENTES}

Como hemos expuesto anteriormente, los docentes decidieron crear un grupo de WhatsApp nuevo. La intención era poder diferenciar las informaciones escolares importantes de aquellas informaciones más personales. Esta intención nos indica que la perspectiva digital democrática promueve que los docentes quieran mejorar la calidad de participación en los espacios digitales de toma de decisiones. En otras palabras, la perspectiva fomenta que los docentes apuesten por mejorar la calidad de acceso a la tecnología digital escolar (Van Dijk, 2005) y mejorar su participación e implicación en las decisiones escolares.

Un segundo resultado que encontramos en las relaciones de participación digital de los docentes es que la perspectiva digital democrática modifica las relaciones de poder que existen en el claustro de profesores. La decisión de crear y diseñar un nuevo grupo de WhatsApp se tomó a partir del interés y la necesidad de todo el claustro de profesores (y no solo desde el equipo directivo). Esta situación nos muestra que esta perspectiva genera experiencias de igualdad entre los docentes, independientemente del cargo que ocupan.

\subsubsection{RELACIONES DE PARTICIPACIÓN DIGITAL DEL ALUMNADO}

A través de los resultados de la investigación sobre las relaciones de participación digital de los alumnos, observamos que al final del proyecto se han producido algunos cambios en la toma de decisiones y el acceso del alumnado.

Un ejemplo es que, al inicio de la investigación, los alumnos podían tomar algunas decisiones sobre aspectos vinculados al aula (elegir con quién hacer los trabajos, con quien sentarse o qué hacer en los ratos libres), pero ninguna decisión vinculada al ámbito digital. En cambio, en los resultados finales, la voz del alumnado (sus opiniones y motivaciones) tomó una nueva dimensión e incidió en el tipo de actividades digitales que se desarrollaban en el aula. Este cambio se produjo porqué los docentes reconocieron la opinión del alumnado y decidieron incorporar sus motivaciones en la práctica educativa del aula. Esta situación la podemos observar en las actuaciones de la recomendación de libros a través de Youtube y de la formación sobre seguridad en la red.

De estos ejemplos destacamos tres aspectos. En primer lugar, observamos que adoptar la perspectiva digital democrática promueve que la escuela busque nuevas formas de articular la participación del alumnado (Dahl, 1999; Dewey, 1995; Feu et al., 2013) y mejore el acceso motivacional a la tecnología (Van Dijk, 2005). Como también, en segundo lugar, promueve que los docentes construyan estrategias para garantizar el mismo acceso competencial a la tecnología digital a todo el alumnado (Van Dijk, 2005) -cuando se ofrece una formación sobre seguridad digital a todo el alumnado-. 
No obstante, en tercer lugar, aunque observamos que la perspectiva digital democrática ha propiciado el reconocimiento de los intereses del alumnado y ha impulsado ciertos cambios en la práctica de aula, aún queda camino por recorrer en lo referente a la toma de decisiones. A través de los resultados observamos que quién acaba tomando la última decisión sobre las actuaciones digitales que conciernen al alumnado son los docentes, y en ningún caso son los propios alumnos. En la fase inicial el alumnado formuló un listado de mejoras que harían en relación con la tecnología digital escolar (por ejemplo, crear un espacio de comunicación web, hacer vídeos sobre sus habilidades, acceder a los ordenadores en espacios informales, entre otros), pero los docentes fueron quienes priorizaron y discriminaron las propuestas, y establecieron como y cuando se llevarían a cabo. Dicho de otra manera, la perspectiva digital democrática favorece el reconocimiento de los intereses y necesidades del alumnado (qué), pero parece que no lo hace partícipe en la toma de decisiones (cómo y cuándo), ni modifica profundamente las relaciones de poder establecidas entre docentes y alumnado (Dijk, 2005; Feu et al., 2013).

Otro aspecto que nos muestran los resultados de investigación vinculado a las relaciones de poder es en cuanto al grado de institucionalización. Observamos que después de adoptar la perspectiva democrática, las familias no sólo toman decisiones en los órganos de gobierno formales (consejo escolar, AMPA, coordinaciones...), sino que se empiezan a tomar en espacios más informales y digitales (el grupo de WhatsApp de familias y docentes). Este espacio digital informal pasa también a ser un espacio de consenso y de toma de decisiones, un espacio de gobernanza.

En los resultados finales también observamos que las familias, aparte de ser las destinatarias de las informaciones escolares, también pasan a tener un papel más activo y participativo (en el momento que toman el cargo de los grupos de WhatsApp y sus normas). Observamos que los docentes les ceden cierto poder de decisión en este ámbito. Esto nos indica que la perspectiva digital democrática condiciona el grado de toma de decisiones, permitiendo a las familias dejar de ser meros receptores de la información y dejándoles formar más parte del ámbito digital escolar.

Los cambios producidos en las relaciones digitales entre familias y escuela, en el grado de institucionalización y en el grado en la toma de decisiones, nos indican que la perspectiva digital democrática incide, de nuevo, en las relaciones de poder (se establecen relaciones más horizontales y de igualdad entre familias y docentes) (Collet-Sabé \& Martori, 2018); en la distribución de recursos (se crean más espacios para comunicar, colaborar y participar) y en la participación digital (Van Dijk, 2005), aunque son los docentes los últimos agentes en aprobar (o no) una decisión.

En relación con el acceso digital, observamos que la perspectiva digital democrática ha impulsado prácticas digitales inclusivas que han perseguido reducir la brecha digital existente entre las familias. Un ejemplo de ello han sido las clases de lengua catalana en la tecnología digital escolar, las cuales han procurado ofrecer a las familias tanto competencias lingüísticas como competencias digitales, para así poderse desenvolver satisfactoriamente en la cotidianidad escolar, independientemente de sus características categóricas (Van Dijk, 2005) -cuando se ofrece una formación sobre seguridad digital a todo el alumnado-.

No obstante, en tercer lugar, aunque observamos que la perspectiva digital democrática ha propiciado el reconocimiento de los intereses 
del alumnado y ha impulsado ciertos cambios en la práctica de aula, aún queda camino por recorrer en lo referente a la toma de decisiones. A través de los resultados observamos que quién acaba tomando la última decisión sobre las actuaciones digitales que conciernen al alumnado son los docentes, y en ningún caso son los propios alumnos. En la fase inicial el alumnado formuló un listado de mejoras que harían en relación con la tecnología digital escolar (por ejemplo, crear un espacio de comunicación web, hacer vídeos sobre sus habilidades, acceder a los ordenadores en espacios informales, entre otros), pero los docentes fueron quienes priorizaron y discriminaron las propuestas, y establecieron como y cuando se llevarían a cabo. Dicho de otra manera, la perspectiva digital democrática favorece el reconocimiento de los intereses y necesidades del alumnado (qué), pero parece que no lo hace partícipe en la toma de decisiones (cómo y cuándo), ni modifica profundamente las relaciones de poder establecidas entre docentes y alumnado (Dijk, 2005; Feu et al., 2013).

\subsubsection{RELACIONES DE PARTICIPACIÓN DIGITAL DE LAS FAMILIAS}

Cuando contrastamos los resultados iniciales y finales sobre la gobernanza escolar de las familias, observamos que después de la investigación-acción se produjeron ciertos cambios en las relaciones de participación digital de las familias. Algunos de los cambios más relevantes están vinculados, por un lado, a las relaciones de poder $y$, por otro lado, al acceso digital.

Vinculado a las relaciones de poder, en los primeros resultados las familias mostraron un alto interés en estar más informadas sobre la cotidianidad escolar y en mejorar su participación en la escuela. De forma similar lo mostra- ban los docentes. Ante esta situación familias y docentes se organizaron para pensar de qué manera podían mejorar su relación y colaboración. Para dar respuesta a esta necesidad crearon conjuntamente un grupo de WhatsApp compartido. Esta situación nos muestra que acoger la perspectiva digital democrática permite a las familias y los docentes hablar, poner en común y reflexionar sobre sus relaciones y cómo participan en el centro, como también les motiva a impulsar actuaciones de mejora. Esta situación nos señala que la perspectiva digital democrática propicia el acercamiento, el reconocimiento y la colaboración entre familias y docentes (Feu et al., 2013).

Otro aspecto que nos muestran los resultados de investigación vinculado a las relaciones de poder es en cuanto al grado de institucionalización. Observamos que después de adoptar la perspectiva democrática, las familias no sólo toman decisiones en los órganos de gobierno formales (consejo escolar, AMPA, coordinaciones...), sino que se empiezan a tomar en espacios más informales y digitales (el grupo de WhatsApp de familias y docentes). Este espacio digital informal pasa también a ser un espacio de consenso y de toma de decisiones, un espacio de gobernanza.

En los resultados finales también observamos que las familias, aparte de ser las destinatarias de las informaciones escolares, también pasan a tener un papel más activo y participativo (en el momento que toman el cargo de los grupos de WhatsApp y sus normas). Observamos que los docentes les ceden cierto poder de decisión en este ámbito. Esto nos indica que la perspectiva digital democrática condiciona el grado de toma de decisiones, permitiendo a las familias dejar de ser meros receptores de la información y dejándoles formar más parte del ámbito digital escolar. 
Los cambios producidos en las relaciones digitales entre familias y escuela, en el grado de institucionalización y en el grado en la toma de decisiones, nos indican que la perspectiva digital democrática incide, de nuevo, en las relaciones de poder (se establecen relaciones más horizontales y de igualdad entre familias y docentes) (Collet-Sabé and Martori, 2018); en la distribución de recursos (se crean más espacios para comunicar, colaborar y participar) y en la participación digital (Van Dijk, 2005), aunque son los docentes los últimos agentes en aprobar (o no) una decisión.

En relación con el acceso digital, observamos que la perspectiva digital democrática ha impulsado prácticas digitales inclusivas que han perseguido reducir la brecha digital existente entre las familias. Un ejemplo de ello han sido las clases de lengua catalana en la tecnología digital escolar, las cuales han procurado ofrecer a las familias tanto competencias lingüísticas como competencias digitales, para así poderse desenvolver satisfactoriamente en la cotidianidad escolar, independientemente de sus características categóricas (Van Dijk, 2005).

\subsubsection{RELACIONES DE PARTICIPACIÓN DIGITAL DEL PAS}

En relación con el PAS, los resultados de la investigación nos muestran que la perspectiva digital democrática promueve, como ya hemos comentado en anteriores situaciones, la creación de nuevos espacios de participación digital, sobre todo cuando la intención es visibilizar aquellos agentes escolares más invisibles (Barroso, 1995; Feu et al., 2013). Sin embargo, tras el análisis de resultados, observamos que la creación de estos espacios digitales ha generado cierto descontento en el PAS que ha participado en la investigación. El análisis de datos nos muestra que este descontento surge porque la incorporación de dicha perspectiva se ha realizado desde la imposición y no desde la propia motivación de los agentes implicados (del equipo directivo al PAS).

Esta situación nos muestra dos aspectos. Por un lado, que la perspectiva digital democrática insiste en reconocer e incluir a aquellos agentes escolares más invisibilizados. Y, por otro lado, que la imposición de la perspectiva digital democrática no garantiza que los agentes participen de una forma real y significativa. Esto hace necesario que, para incorporar dicha perspectiva de forma satisfactoria, sea necesario mejorar las relaciones de poder, fomentar la equidad en la distribución de recursos (Van Dijk, 2005) e incorporar dicha perspectiva desde la propuesta, la construcción colaborativa y la toma de decisiones conjunta (Feu et al., 2013).

\section{CONCLUSIONES}

A través de los resultados de la investigación-acción podemos extraer tres grandes conclusiones. En primer lugar, podemos concluir que la perspectiva digital democrática empuja a los diferentes miembros de la comunidad escolar: a hablar conjuntamente sobre participación escolar; reflexionar sobre cuáles son las relaciones de poder existentes en el centro; repensar la distribución de recursos digitales; y mejorar el acceso digital escolar.

En segundo lugar, observamos que la perspectiva digital democrática promueve, por un lado, el reconocimiento de todos los miembros de la comunidad educativa en los órganos de toma de decisiones. Y, por otro lado, fomenta la generación de nuevos espacios digitales de participación más inclusivos, y menos generadores de desigualdad. 
En tercer y último lugar, pese a las claras intenciones de la perspectiva en mejorar la calidad digital y democrática, observamos que aún existe un orden jerárquico que limita el avance hacia una calidad óptima. Un orden en el cual los docentes son los que tienen la última palabra (el poder) en todo lo referente a la tecnología digital escolar y la participación (sobre el alumnado, sobre las familias y sobre el PAS). Los docentes son los últimos responsables en decidir qué necesidades se priorizan, qué ac- tuaciones se desarrollan y como se debe hacer. En este sentido, aún queda camino por recorrer y esta perspectiva debe ir avanzando para lograr una mayor horizontalidad y una mejor participación e inclusión de todos y cada uno de los agentes escolares. Y de este modo, evitar posibles situaciones de exclusión (como en el caso de la priorización del listado de necesidades del alumnado) o de descontento (como el que hemos observado en caso del PAS).

\section{REFERENCIAS}

Adell, J., \& Castañeda, L. (2015). Las pedagogías escolares emergentes. Cuadernos de Pedagogía, (462), 21-25.

Albar, E. (1996). La vida social de las máquinas: orígenes, desarrollo y perspectivas actuales en la sociología de la tecnología. Reis: Revista Española de Investigaciones Sociológicas, (76), 141-172. https://doi. org/10.2307/40183990

Baena, S., Collet-Sabé, J., Garcia-Molsosa, M., \& Manzano, M (2020). More innovation, less inclusion? Debates and discussions regarding the intersectionality of innovation and inclusion in the Catalan school system: a position paper. International Journal of Inclusive Education, https://doi.or g/10.1080/13603116.2020.1736653

Barroso, J. (1995). Para o desenvolvimento de uma cultura de participação na escola. Cadernos de Organização e Gestão Escolar, (1).

Bartolomé, M. (1992). Investigación cualitativa en educación ¿Comprender o transformar? Revista de Investigación Educativa, 20(2), 7-36.

Beneyto-Seoane, M., \& Collet-Sabé J. (2016). Las relaciones digitales entre familias y escuela: análisis y propuestas. @tic. Revista d'innovació educativa, 16, 1-9. https://doi: 10.7203/attic.16.6894.

Beneyto-Seoane, M., \& Collet-Sabé J. (2018). Análisis de la actual formación docente en competencias TIC. Por una nueva perspectiva basada en las competencias, las experiencias y los conocimientos previos de los docentes. Profesorado: revista de currículum y formación del profesorado, 22(4), 91-110.

Beneyto-Seoane, M., Collet-Sabé, J., Cortada-Pujol, M., \& Sánchez-Garrote, I. (2013). ¿Mucho ruido y pocas nueces? Escuelas, familias y TIC; discursos, realidades, logros y retos. In A. Daniel, A. Creus, y J. Sánchez-Navarro (Eds.), Educación, medios digitales y cultura de la participación ( $p$. 241-262). UOC Press. 
Beneyto-Seoane, M., Castillo, J., Collet-Sabé, J., \& Tort, A. (2019). Can schools become an inclusive space shared by all families? Learnings and debates from an action research project in Catalonia. Educational Action Research, 27(2), 210-226.

Bisquerra, R. (2004). Metodología de la investigación educativa. La Muralla.

Bosco, A., Sánchez-Valero, J.A., \& Sancho-Gil, J. M. (2016). Teaching practice and ICT in Catalonia: Consequences of educational policies. KEDI Journal of Educational Policy, 13(2), 201-220.

Castells, M. (2003). La interacció entre les Tecnologies de la Informació i la Comunicació i la Societat Xarxa: un procés de canvi històric. Coneixement i Societat: Revista d'Universitats, Recerca i Societat de La Informació, (1), 8-21.

Cobo, C. (2017). La promesa digital y la herencia analógica en la educación. In P. Rivera-Vargas, E. Sánchez-Sánchez, R. Morales-Olivares, I. Sáez-Rosenkranz, C. Yévenes, \& S. Butendieck (Eds.), Conocimiento para la equidad social: pensando Chile Globalmente (pp. 117-124). Colección Políticas Públicas - USACH.

Cohen, L., Manion, L., \& Morrison, K. (2011). Research methods in education. Routledge.

Collet-Sabé, J., \& Martori, J.C. (2018). Bridging boundaries with Bernstein: approach, procedure and results of a school support project in Catalonia. British Journal of Sociology of Education, 39(8), 1126-1142.

Dahl, R. A. (1999). La democracia: una guía para los ciudadanos. Taurus.

Dewey, J. (1995). Democracia y educación: una introducción a la filosofía de la educación. Morata.

Elliott, J. (1993). El cambio educativo desde la investigación-acción. Ediciones Morata.

Ferrance, E. (2000). Action Research. LAB at Brown University.

Feu, J., Serra, C., Canimas, J., Làzaro, L., \& Simó-Gill, N. (2017). Democracy and Education: A Theoretical Proposal for the Analysis of Democratic Practices in Schools. Studies in Philosophy and Education, 36, 647-661. https://doi.org/10.1007/s11217-017-9570-7

Feu, J., Serra, C., Canimas, J., \& Simó, N. (2013). El concepto teórico de democracia aplicado a la educación: modelos teóricos y consecuencias de los mismos. En Comunicación presentada al XI Congreso Español de Sociología (p. 25). Madrid.

Feu, J., Simó, N., Serra, C., \& Canimas, J. (2016). Dimensiones, características e indicadores para una escuela democrática. Estudios Pedagógicos 42(3), 449-465. https://doi.org/10.4067/S0718-07052016000400024 
Fullan, M. (2013). Stratosphere: Integrating technology, pedagogy and change knowledge. Pearson.

Kemmis, S., \& MacTaggart, R. (1988a). Cómo planificar la investigación-acción. Laertes.

Kemmis, S., \& McTaggart, R. (1988b). The Action research planner. Deakin University.

Latorre, A. (2003). La Investigación-acción: conocer y cambiar la práctica educativa. Graó.

Lewin, K. (1946). Action research and minority problems. Journal of Social Issues, 2(4), 34-46. https://doi.org/10.1111/j.1540-4560.1946.tb02295.x

McNiff, J., Lomax, P., \& Whitehead, J. (2003). You and Your Action Research Project (Second Edition). RoutledgeFalmer.

Quintanal, J., \& García, B. (2012). Fundamentos básicos de metodología de investigación educativa. Editorial CCS.

Rodríguez, G., Gil, J., \& García, E. (1999). Metodología de la investigación cualitativa. Ediciones Aljibe.

Selwyn, N. (2011). Schools and schooling in the digital age: a critical analysis. Routledge.

Selwyn, N. (2016). Is technology good for education? Polity.

Stenhouse, L. (1993). La investigación como base de la enseñanza. Ediciones Morata.

Ulvik, M., Riese, H., \& Roness, D. (2017). Action research - connecting practice and theory. Educational Action Research, 1-15. https://doi.org/10.1080/ 09650792.2017 .1323657

Van Deursen, A. J. A. M., \& Van Dijk, J. A. G. M. (2010). Measuring Internet Skills. International Journal of Human-Computer Interaction, 26(10), 891-916. https://doi.org/10.1080/10447318.2010.496338

Van Dijk, J. A. G. (2012). The Evolution of the Digital Divide. The Digital Divide turns to Inequality of Skills and Usage. En J. Bus, M. Crompton, M. Hildebrandt, \& G. Metakides (Ed.), Digital Enlightenment Yearbook (pp. 57-78). IOS Press. https://doi.org/10.3233/978-1-61499-057-4-57

Van Dijk, J. A. G. M. (2005). The deepening divide: inequality in the information society. Sage Publications.

Van Dijk, J. A. G. M., \& Van Deursen, A. J. A. M. (2014). Digital skills: unlocking the information society. Palgrave Macmillan US. 